\title{
Kynurenines increase MRS metabolites in basal ganglia and decrease resting-state connectivity in frontostriatal reward circuitry in depression
}

\author{
Xiangchuan Chen (iD ${ }^{1,2}$, Diana J. Beltran (iD ${ }^{1,2}$, Valeriya D. Tsygankova (iD ${ }^{1,2}$, Bobbi J. Woolwine ${ }^{1,2}$, Trusharth Patel (D) ${ }^{3}$, Wendy Baer ${ }^{1,2}$, \\ Jennifer C. Felger ${ }^{1,2}$, Andrew H. Miller (iD ${ }^{1,2}$ and Ebrahim Haroon (iD) ${ }^{1,2}$
}

(C) The Author(s) 2021

Inflammation is associated with the development of anhedonia in major depression (MD), but the pathway by which inflammatory molecules gain access to the brain and lead to anhedonia is not clear. Molecules of the kynurenine pathway (KP), which is activated by inflammation, readily influx into the brain and generate end products that alter brain chemistry, disrupt circuit functioning, and result in the expression of inflammatory behaviors such as anhedonia. We examined the impact of plasma and CSF KP metabolites on brain chemistry and neural function using multimodal neuroimaging in 49 depressed subjects. We measured markers of glial dysfunction and distress including glutamate (Glu) and myo-inositol in the left basal ganglia using magnetic resonance spectroscopy (MRS); metrics of local activity coherence (regional homogeneity, ReHo) and functional connectivity from resting-state functional MRI measures; and anhedonia from the Inventory for Depressive Symptoms-Self Report Version (IDS-SR). Plasma kynurenine/tryptophan (KYN/TRP) ratio and cerebrospinal fluid (CSF) 3-hydroxykynurenine (3HK) were associated with increases in left basal ganglia myo-inositol. Plasma kynurenic acid (KYNA) and KYNA/QA were associated with decreases and quinolinic acid (QA) with increases in left basal ganglia Glu. Plasma and CSF KP were associated with decreases in ReHo in the basal ganglia and dorsomedial prefrontal regions (DMPFC) and impaired functional connectivity between these two regions. DMPFC-basal ganglia mediated the effect of plasma and CSF KP on anhedonia. These findings highlight the pathological impact of KP system dysregulation in mediating inflammatory behaviors such as anhedonia.

Translational Psychiatry (2021)11:456; https://doi.org/10.1038/s41398-021-01587-1

\section{INTRODUCTION}

Previous data has suggested that one mechanism by which inflammation can influence behavior is through effects on brain glutamate (Glu). Chronic administration of the inflammatory cytokine interferon (IFN)-alpha in patients with hepatitis $C$ led to increased Glu in basal ganglia regions and anterior cingulate cortex measured using magnetic resonance spectroscopy (MRS) $[1,2]$. Similar increases in basal ganglia Glu have also been reported in patients with major depression (MD) with increased endogenous inflammation, indexed by the acute phase reactant C-reactive protein (CRP) [3]. In each case, increased Glu in these brain regions was associated with increases in the overall severity of depressive symptoms and symptoms of anhedonia, amotivation, and psychomotor slowing [1-3]. Of note, anhedonia is rapidly emerging as a public health priority [4]. For instance, anhedonia is not only common in MD (rates of $\sim 70 \%$ ) but was associated with a $\sim 30 \%$ increase in the risk of death and disability among depressed individuals [5]. Large-scale meta-analyses and reviews have linked the development of anhedonic symptoms with basal ganglia and related reward network dysfunction [6-8]. Increasing inflammation of the glial cells in the basal ganglia and related regions may account for reward circuit dysfunction and anhedonia in the inflammatory subtype of $\mathrm{MD}[3,9,10]$.

Astrocytes are primarily responsible for Glu uptake in synaptic and nonsynaptic areas and, consequently, are the major regulators of Glu homeostasis [11]. Astroglial dysfunction is a welldocumented feature of mood disorders but may be particularly relevant to the inflammatory subtype of $\operatorname{MD}[12,13]$. MRS Glu and myo-inositol are believed to index astroglial dysfunction and distress $[14,15]$. Despite its presence in other tissues, myo-inositol is believed to be an astroglial marker, because it is highly concentrated in astroglial cells, as shown in in vivo NMR studies $[14,15]$. Myo-inositol participates in astrocytic osmoregulation and is highly sensitive to inflammation and oxidative stress [14-16]. Accordingly, greater CRP concentrations in the cerebrospinal fluid (CSF) of MD patients were associated with increases in myoinositol, a marker of astroglial distress in the basal ganglia, suggesting increased astroglial distress [3, 17].

Relevant to the impact of inflammation on Glu and astrocytic function, there has been an increasing interest in the potential role of metabolites of the kynurenine pathway (KP). Inflammatory stimuli such as lipopolysaccharide (LPS) and Bacillus

${ }^{1}$ Emory Behavioral Immunology Program, Atlanta, GA, USA. ${ }^{2}$ Department of Psychiatry and Behavioral Sciences, Atlanta, GA, USA. ${ }^{3}$ Department of Anesthesiology, Emory University School of Medicine, Atlanta, GA, USA. ${ }^{\circledR}$ email: eharoon@emory.edu

Received: 16 May 2021 Revised: 4 August 2021 Accepted: 19 August 2021

Published online: 04 September 2021 
Calmette-Guérin (BCG) vaccine and inflammatory cytokines such as tumor necrosis factor (TNF) and IFN gamma activate the enzyme indoleamine 2,3 dioxygenase (IDO), which converts tryptophan (TRP) to kynurenine (KYN) [18-20]. Under homeostatic conditions, $\sim 95 \%$ of TRP is converted into KYN, and its breakdown products culminating in the generation of nicotinamide adenine dinucleotide (NAD+), an important cellular energy source [21, 22]. $\mathrm{KYN}$ is then transported into the brain, where it is metabolized either into 3-hydroxykynurenine (3HK), anthranilic acid (AA), 3-hydroxyanthranilic acid (3HAA), and quinolinic acid (QA) in microglia, tissue macrophages, and trafficking monocytes; or kynurenic acid (KYNA) in astrocytes, oligodendrocytes, and neurons [23-27]. KYN and 3HK gain access to the brain via the large neutral amino acid transporter (LAT-1) in the blood-brain barrier (BBB), leading to an excess of KP metabolism in the brain [28-30]. These data are consistent with findings in patients with MD, whereby path analyses have demonstrated that increased peripheral blood inflammatory markers are linked to increased peripheral blood KP metabolites that are in turn associated with increased CSF KP metabolites [31]. Of note, in the context of chronic immune activation, astrocytes generate large quantities of KYN that can be converted into QA by adjacent macrophages and microglia [32]. KYN can also amplify local proinflammatory signaling via activation of the nod-like receptor protein (NLRP) -2 inflammasome complex in astrocytic cells [33].

KP metabolites influence glutamatergic activity in several distinct ways [34]. QA produced endogenously, may be an endogenous neurotoxin and gliotoxin at high concentrations [35]. Regarding the impact of KP metabolites on astrocytic regulation of Glu, QA directly promotes astrocytic Glu release, increases its cycling, and decreases its reuptake by astrocytic transporters [34, 35]. 3HK also increases astrocytic Glu release via the induction of reactive oxygen species, leading to activation of the system $\mathrm{xC}$ - antiporter, which extrudes one molecule of Glu exchange for a molecule of cysteine that is used in the synthesis of the antioxidant glutathione [36, 37]. Another target for KP metabolites is the $\mathrm{N}$-methyl-D-aspartate (NMDA)-Glu receptor on glutamatergic neurons, with QA acting as an agonist and KYNA acting as an antagonist and allosteric modulator at the glycine site [34, 35, 37].

These effects of KP metabolites in conjunction with inflammation on astrocytes and Glu cycling can contribute to excessive Glu release and spillover into the extrasynaptic space $[38,39]$. Furthermore, the diffusion of the spillover Glu in the extracellular fluid leads to chaotic activation of extrasynaptic NMDA receptors leading to disarrayed signaling [40-43]. Of note, patterns of spontaneous brain oxygen level-dependent (BOLD) oscillatory activity in rsfMRI offer an unprecedented opportunity to study chaotic functional brain activity [44-51]. More specifically, regional homogeneity (ReHo) quantifies the concordance in BOLD-oscillations between neighboring voxels. ReHo has been associated with regional pathology in brain disease models [10, 52-54]. We have previously reported that inflammationassociated increases in Glu were associated with decreased ReHo in the basal ganglia and several other brain regions associated with mood and reward processing [10].

Indeed, MD patients with concurrent increases in inflammation and CNS Glu exhibited ReHo decreases at the site of MRS acquisition and over a wider network of brain regions, particularly those involved in reward processing [10]. Impaired connectivity between regions with decreased ReHo was associated with greater depression, anhedonia, and psychomotor slowing [10]. Our previous data and those from other groups have consistently linked concurrent increases in inflammation and KP metabolism with depression, suicidality, anhedonia, and treatment nonresponse [31, 55-59]. These findings are also consistent with a rich literature linking the development of depression-like behavior in preclinical models (e.g., decreased sucrose preference, greater immobility on forced-swim or tail-suspension tests) following IDOactivation by inflammatory stimuli [20, 27, 60-63]. Finally, in laboratory animals, blocking KP activation through inhibition of IDO or inhibiting the transport of KYN into the brain using leucine or blocking of NMDA receptors with ketamine can reverse inflammation-induced depressive-like behavior [20, 30, 61, 63].

Nevertheless, no study has examined the associations of plasma and CSF KP metabolites with MRS and rsfMRI measures (Glu, myoinositol, ReHo, and connectivity). We, therefore, examined the impact of plasma and CSF KP on brain chemistry and the function of basal ganglia using MRS and rsfMRI. We hypothesized that increased plasma or CSF KP measures would be associated with elevated MRS-signals of astroglial markers (Glu and myo-inositol), reduced ReHo, and decreased functional connectivity in BG and other reward-related brain regions, resulting in greater severity of anhedonia. Identifying such a biomarker/neuroimaging-based biosignature could help identify a distinct subgroup of depressed patients with increased inflammation who may respond to KPtargeted pharmacotherapeutics. Subjects presented herein represent a subset of patients recruited for an $\mathrm{NIH}$-funded study on phenotyping depressed patients with increased inflammation and overlap with subjects that have appeared in previous reports (NCT01426997) [3, 10, 31, 64].

\section{PATIENTS AND METHODS \\ Study sample}

We included unmedicated subjects between the ages of 21-65 years with a primary diagnosis of MD based on Structured Clinical Interview for Diagnostic and Statistical Manual-IV (SCID-IV) [65] and a 17-Item Hamilton Rating Scale for Depression score $(H A M-D) \geq 18$ [66]. Subjects with active suicidal ideation (score $>2$ on item \#3 on HAM-D) were excluded from study participation and referred for treatment. All qualifying patients were monitored for safety (e.g., suicidality, worsening) and referred for further management if necessary. We determined study eligibility based on history and physical examination, psychiatric evaluation, laboratory testing, and electrocardiography. Presence of Axis I psychiatric disorders (other than anxiety); substance abuse/dependence within the past 6 months (SCID-IV criteria and urine drug testing); unstable medical disorders (needing multiple visits or medication changes); intake of psychotropic medications of any kind (other than prn benzodiazepines, last drug intake $>72 \mathrm{~h}$ ); or intake of medications known to affect the immune system (oral steroids, nonsteroidal anti-inflammatory agents) were exclusionary. Patients with autoimmune disorders or a history of cancer (other than basal cell skin cancer) were excluded. No individual was allowed to discontinue their current psychotropic medications for the study. The Institutional Review Board of Emory University approved the study, and all subjects provided written informed consent.

\section{Assessment of depression severity and anhedonia}

Standardized measures of depression severity were obtained using the 30item Inventory for the Severity of Depression-Self-Rated Version (IDS-SR)a scale that has been extensively used in previous studies by our and other groups $[3,64,67,68]$. IDS-SR was completed in one sitting, and the patients were asked to choose the item response $(0,1,2$, or 3$)$ that best described themselves over the past 7 days. The scale was scored by adding 28 of 30 items to obtain a total score ranging from $0-84$, excluding items \#11-12 (Appetite increase/decrease), and \#13-14 (Weight increase/decrease)-per instructions from the developers [68]. In addition, items with ambiguous ratings including \#9 ("Diurnal Variation of Mood" with two subitems 9A and 9B), \#10 ("Quality of Mood" with difficulty in differentiating depression versus grief), and \#25 (aches and pains with difficulty in differentiating items from other somatic symptoms) were excluded based on patient feedback. Hierarchical clustering of the remaining 23 individual IDS-SR items indicated that four anhedonia items (\#8: "Response to Good or Desired Events," \#17: "View of My Future," \#19: "General Interest Excluding Sex," and \#22: "Interest in Sex") tightly clustered together. Anhedonia was determined as a latent factor representing the above individual items rather than a composite summary score from specific questionnaires.

\section{Blood sampling and assays}

Blood sampling was conducted between $0800-1000 \mathrm{~h}$, and all subjects sat quietly for 30 min before phlebotomy to minimize the impact of circadian 
variations and stress. The sampled blood was immediately centrifuged at $1000 \mathrm{xg}$ for $10 \mathrm{~min}$ at $4{ }^{\circ} \mathrm{C}$. Plasma was then removed and frozen at $-80^{\circ} \mathrm{C}$ for batched assays. Lumbar puncture (LP) was performed at the Emory General Clinical Research Center between 1100 and $1500 \mathrm{~h}$ by an attending anesthesiologist 1 day after blood sampling, as described previously [31, 69]. Approximately $10 \mathrm{~mL}$ of CSF was collected for each subject after discarding the initial $1 \mathrm{~mL}$ to avoid blood contamination. Samples were collected into chilled tubes, aliquoted into $1 \mathrm{~mL}$ vials, and immediately frozen at $-80^{\circ} \mathrm{C}$ until batched assays.

KP assays. Concentrations of TRP, KYN, KYNA, 3HK, AA, 3HAA, and QA in plasma and the CSF were measured blind to diagnosis by Brains Online, LLC (Charles River, Inc). The serum and CSF metabolite concentrations were estimated using high-performance liquid chromatography (HPLC) with tandem mass spectrometry (MS/MS) detection using standardized protocol per specifications provided by the vendor. The overall assay CV was $<10 \%$ for TRP, KYN, KYNA, AA, 3HAA, 3HK, and QA in the plasma. Supplementary Information 1 and Supplementary Table 1 provides assay reliability data. All assays with unreliable quantitation were excluded. KYN/TRP and QA/KYNA ratios were computed for further analyses.

Immune assays. Plasma concentrations of high-sensitivity CRP were determined by the immunoturbidometric method (Sekisui Diagnostics, Lexington, MA, USA) on a Beckman AU480 automatic analyzer. Cytokines and their receptors were assayed in duplicate or triplicate for plasma and CSF, respectively, using multiplex bead-based assays (R\&D Systems, Minneapolis, MN, USA) and read with a MAGPIX CCD imaging system (Luminex, Austin, TX), as described in previous publications $[64,69]$. Based on our previous work showing a strong relationship with KP metabolites in plasma and CSF, TNF, and TNF receptor type-2 (TNFR2) were measured in plasma and CSF based on specifications detailed in our previous studies [31].

\section{MRS/MRI analysis}

MRI scans were acquired using a Siemens $3 \mathrm{~T}$ Trim-Trio scanner (Siemens Healthineers, Malvern, PA, USA). Head motion was limited with foam restraints. T1 images were acquired using 3D-MPRAGE sequence (TR/TE/ TI $=2300 / 3.02 / 1100 \mathrm{~ms}$, flip angle $\left(F A=80^{\circ}\right.$, voxel size $=1 \times 1 \times 1 \mathrm{~mm}^{3}$, matrix size $=256 \times 256$ ). rsfMRI data were acquired using an EPI sequence $\left(\mathrm{TR} / \mathrm{TE}=2950 / 30 \mathrm{~ms}, \quad \mathrm{FA}=90^{\circ}, \quad \mathrm{FOV}=220 \mathrm{~mm}, \quad\right.$ matrix size $=3.4 \times$ $3.4 \mathrm{~mm}^{3} \times 4.0 \mathrm{~mm}^{3}$, slice number $=30,150$ repetitions). Participants were instructed to keep their eyes open and look at a fixed crosshair on the scanner's projection screen. Single-voxel MRS (MRS-SVS) data were acquired using a PRESS sequence (TR/TE $=3000 / 30 \mathrm{~ms}, \mathrm{FA}=90^{\circ}$, Spectral points $=1024$, size of a voxel of interest [VOI] $=30 \mathrm{~mm} \times 17 \mathrm{~mm} \times 17 \mathrm{~mm}$ [3], 128 averages with water suppression, four with water unsuppressed scans). The VOI was placed in the left basal ganglia region, as described in our previous publication $[3,10]$. The data were preprocessed by correcting signal spikes, slice-timing shift, and head motion, and removing motion and CSF signals with a general linear model (GLM). The residual time-series data were used to calculate ReHo, converted into the MNI space, and spatially smoothed (full-width half-maximum, FWHM $=4 \mathrm{~mm}$ ). KP-related brain regions of interest (ROIs) were identified by correlating the ReHo with KP measures. The analysis was conducted using recommended clusterwise multiple-test correction (voxel-level $p<=0.001$ and cluster-wise $p<$ $=0.05)[70,71]$. ReHo-KP marker correlations were limited to effects found significant in the KP-MRS analysis to curtail multiple comparisons. KPrelated ReHo ROls thus identified were set as seeds for the connectivity analysis. After further removal of the WM signals and temporal filtering $(0.01-0.1 \mathrm{~Hz})$, averaged time-series data was calculated for each ReHo ROI and then correlated with the time-series data of individual voxels in the brain. Connectivity data from the CSF KP ReHo ROls were correlated with the CSF KP measures, and those from the plasma KP ReHo ROls were correlated with the plasma KP measures. Individual CSF and plasma KP measures were used independently to define both the seed (ReHo ROIs) and its target connectivity ROls after applying the abovementioned multiple correction threshold. Partial-volume correction of absolute quantitation of metabolite concentrations from MRS was performed based on the volumetric ratios of GM, WM, and CSF in the VOI, as described in our earlier publications [3, 10]. See Supplementary Information 2 for more details of MRI data acquisition and analysis.

\section{Statistical analysis-Lasso-inferential model}

Penalizing, cross-fit, partialing out (double machine learning), lassoinferential models robust to collinearity were used in Stata v16 (Stata,
College Station, TX, USA) to examine the association between MRS markers and KP metabolites after controlling for age, sex, race (Caucasian vs. African American), and body mass index (BMI). Independent models for each of the MRS markers (Glu and myo-inositol) and KP metabolites from each compartment (plasma and CSF) were used after partialing out effects of covariate/control variables (age, sex, BMI, and race) per Frisch-Lovell-Waugh theorem. The analysis was geared to test the explanatory power of the primary variables of interest (primary model) and to examine the effect of adding or removing covariates upon these associations (auxiliary model). We conducted this analysis in two phases. The primary model used a plugin estimator consisting of $\mathrm{k}(10)-$ fold*resample(10) runs (total $=100$ runs), and the secondary (sensitivity) analysis used alternate estimators, including cross-validation (CV) and adaptive methods. Of note, noise factors induced by control covariates do not disrupt the primary (plugin) estimator than the auxiliary (crossvalidated or adaptive) estimators. Partial least squares-structural equation modeling (PLS-SEM) was used to examine path effects and mediational associations in Smart PLS [72]. Biomarkers (KP, immune), seed connectivities, and anhedonia items (of IDS-SR) were used as indicators to generate latent constructs (measurement model) that were further used to test hypotheses based on path associations (structural model). Moderation of these associations by covariates was tested using linear path models for continuously distributed variables (age, BMI) and using group comparison models for categorical covariates (sex, race). More details of the analysis plan are provided in Supplementary Information 3.

\section{RESULTS}

\section{Background data}

Forty-nine subjects with available KP and MRS measures were recruited, of whom 46 subjects with available plasma KP and MRS measures and 40 with KP and rsfMRI measures were included in the study. Details on sample availability is provided in Supplementary Information 4, and missing data information are provided in Supplementary Table 2. Background and clinical information are provided in Table 1. None of the missing biomarker data were imputed as it was adjudged that the missingness was not random. One subject did not answer item \#22 on IDS-SR (item on Libido), and the result was imputed using low-rank approximation after verifying it was missing completely at random. The subjects were in moderate to severely depressed range based on HAM-D Scores (mean \pm SD $=23.2 \pm 3.2$ ), aged between 21-64 (mean $\pm \mathrm{SD}=38.6 \pm 10.9$ years), and obese $\left(B M I=31.8 \pm 7.9 \mathrm{~kg} / \mathrm{m}^{2}\right) .69 \%$ of the subjects were women, and African Americans comprised $65 \%$ of the study population. The high percentage of women is a characteristic of most depressed subject groups, and the high representation of African Americans is consistent with the study location (Atlanta, GA). Several values of CSF KYNA, QA, and 3HAA were discarded due to estimation difficulties by BrainsOnline, Inc. Hence only 4 CSF KP metabolites (TRP, KYN, 3HK, and AA) and one ratio (CSF KYN/TRP) were available for analysis.

\section{Plasma KYNA, QA, and KYNA/QA association with MRS Glutamate}

Using the cross-fit, partialing out lasso, plasma QA (Fig. 1A) was positively $(\mathrm{Cf}=0.53,95 \% \mathrm{Cl}=(0.07,0.99), z=2.41, p=0.024)$ and plasma KYNA (Fig. 1B) was negatively $(\mathrm{Cf}=-0.40,95 \% \mathrm{Cl}=$ $(-0.74,-0.05), z=-2.25, p=0.02)$ associated with left basal ganglia Glu. The associations survived multiple testing by using 100 -fold resampling of each fold in the primary analysis and during the sensitivity analysis, indicating relative insensitivity to perturbation by covariate induced noise factors. None of the other metabolites were significantly associated with MRS markers. Plasma KYNA/QA ratio was negatively associated with left basal ganglia Glu (Fig. 1C, Cf(95\% Cl) $=-0.40(-0.63,-0.16), z=-3.27$, $p=0.001)$. The relationship between plasma KYNA, QA, KYNA/QA was robust (survived 100 -fold resampling) and was not perturbed by adding or removing covariate/control variables during sensitivity analysis using CV and adaptive estimations. 
Table 1. Demographic, clinical, and biological variables of the sample.

\begin{tabular}{|c|c|c|c|c|c|}
\hline Variable & $N$ & (\%) & Mean(Std Dev) & Min & Max \\
\hline Age $(y)$ & 49 & & $38.6(10.9)$ & 21 & 64 \\
\hline Female & 34 & $69 \%$ & & & \\
\hline Male & 15 & $31 \%$ & & & \\
\hline Caucasian & 17 & $17 \%$ & & & \\
\hline African American & 32 & $65 \%$ & & & \\
\hline BMI $\left(\mathrm{kg} / \mathrm{m}^{2}\right)$ & 49 & & $31.8(7.9)$ & 19.6 & 52.3 \\
\hline HAM-D & 49 & & $23.2(3.2)$ & 18 & 32 \\
\hline LBG Glu (mmol/kg) & 49 & & $8.7(1)$ & 6.4 & 10.8 \\
\hline $\begin{array}{l}\text { LBG myo-inositol } \\
(\mathrm{mmol} / \mathrm{kg})\end{array}$ & 49 & & $4.9(0.9)$ & 3.5 & 7.4 \\
\hline Plasma TNF (pg/ml) & 49 & & $5.5(2.2)$ & 0.1 & 10.6 \\
\hline Plasma TNFR2 (ng/ml) & 49 & & $2.5(0.9)$ & 0.8 & 5.3 \\
\hline Plasma TRP $(\mu \mathrm{M})$ & 49 & & $50.6(8.7)$ & 35 & 73.4 \\
\hline Plasma KYN ( $\mu \mathrm{M})$ & 49 & & $1.6(0.4)$ & 0.9 & 3.3 \\
\hline Plasma KYNA (nM) & 48 & & $37.4(17.7)$ & 13.2 & 101 \\
\hline Plasma AA (nM) & 47 & & $6.1(2.4)$ & 2.6 & 13.8 \\
\hline Plasma 3HK (nM) & 49 & & $32.1(11.4)$ & 12.5 & 72.5 \\
\hline Plasma 3HAA (nM) & 47 & & 20.4(9.4) & 8.8 & 51.4 \\
\hline Plasma QA (nM) & 49 & & $432.8(140)$ & 184 & 733 \\
\hline CSF TRP $(\mu \mathrm{M})$ & 41 & & $1.6(0.4)$ & 0.5 & 2.5 \\
\hline CSF KYN (nM) & 41 & & $32.4(15.6)$ & 13.7 & 96.2 \\
\hline CSF KYNA (nM) & 36 & & $1.5(0.7)$ & 0.7 & 3.7 \\
\hline CSF AA (nM) & 42 & & $2.5(0.8)$ & 1.3 & 5 \\
\hline CSF 3 HKYN (nM) & 42 & & $4.8(5.2)$ & 1.3 & 21.4 \\
\hline CSF QA (nM) & 37 & & $20.1(10.6)$ & 10.5 & 56.7 \\
\hline
\end{tabular}

The table presents the background data of the sample.

$B M I$ body mass index, HAM-D 17-item Hamilton depression rating scale, $L B G$ left basal ganglia, Glu glutamate, TNF tumor necrosis factor, TNFR2 tumor necrosis factor receptor type 2, TRP tryptophan, KYN kynurenine, KYNA kynurenic acid, $A A$ anthranilic acid, $3 H K$ 3-hydoxykynurenine, $3 H A A$ 3-hydoxyanthranilic acid, QA quinolinic acid, CSF cerebrospinal fluid.

\section{Plasma KYN/TRP and CSF 3HK associations with left basal ganglia myo-inositol}

Plasma KYN/TRP ratio was positively associated with left basal ganglia myo-inositol $(\mathrm{Cf}(95 \% \mathrm{Cl})=0.25(0.02,0.48), z=2.17, p=$ 0.03 ) only in the primary model. This association did not survive sensitivity analysis due to a strong interaction between myoinositol and age. The potent oxidative stress-inducing molecule CSF 3HK (Fig. 1D) was associated with left basal ganglia myoinositol $(\mathrm{Cf}(95 \% \mathrm{Cl})=0.45(0.19,0.75), z=3.35, p=0.001)$ in the primary model after 100 -fold repetitions. However, this association did not survive sensitivity analysis as age was independently associated with myo-inositol, while BMI impacted CSF 3HK. None of the other CSF KP makers or CSF KYN/TRP ratio were associated with MRS markers. The inset in Fig. $2 \mathrm{~A}$ illustrates the MRI voxel used for acquisition.

$\mathrm{KP}$ associations with rsfMRI measures (Fig. 2A): Based on the above results, only KYN, 3HK, KYNA, QA, KYN/TRP, and KYNA/QA ratios in the plasma and KYN and $3 \mathrm{HK}$ in the CSF were used for ReHo analysis. Plasma KYNA, QA, and KYNA/QA yielded ReHo ROIs identical to those provided by KYN and $3 \mathrm{HK}$ (provided in Supplementary Information 5). Accordingly, ReHo data correlated with plasma KYN, plasma 3HK, CSF KYN, and CSF $3 \mathrm{HK}$ were used from all 40 subjects. ReHo ROI correlated with the above four markers were used as originating seeds to identify altered connectivity between these and other brain regions as a function of the four KP metabolites. After adjusting for multiple comparisons (voxel $p<=0.001$, cluster $p<=0.05$ ), the $4 \mathrm{KP}$ measures were associated with decreased ReHo in a total of 12 ROIs. Besides, 19-decreased seed connectivities between these ROls and other brain regions were also noted. Supplementary Tables 3, 4 and Supplementary Figs. 1-3 provides a full list of ReHo ROls and seed connectivities related to the KP metabolites. In terms of voxel volume, ReHo decreases in the DMPFC and basal ganglia together accounted for $62 \%$ of total brainwide ReHo decreases identified by all four KP metabolites (Fig. 2B). Additionally, $90 \%$ of seed-connectivity decreases explained by the above four KP measures also involved either DMPFC or basal ganglia ROIs (Fig. 2C).

CSF KP mediates the effects of plasma KP on DMPFC-basal ganglia connectivity (Fig. 2D). PLS-SEM was used for path analysis using Baron and Kenny's four-step approach [73]. The mediation model included three factors, i.e., plasma KP- and CSF KP-factors (latent factors generated from KYN and 3HK from plasma and CSF, respectively), and DMPFC-basal ganglia connectivity factor correlated with metabolites used to derive CSF KP latent (including DMPFC-left basal ganglia, DMPFC-right basal ganglia, left basal ganglia-left basal ganglia, and left basal ganglia-right basal ganglia). All values reported in the path modeling were obtained using 10000-bootstrapped repetitions. Step 1 (path "c") examined if plasma KP latent (predictor variable) was associated with DMPFC-basal ganglia connectivity latent. Plasma KP factor was associated with DMPFC-basal ganglia factor with a negative path $(\mathrm{Cf}(95 \% \mathrm{Cl})=-0.56(-0.78,-0.37), t=3.36, p=0.001)$ with a strong effect size $(r$-sq $=0.31$, Cohen's $f$-sq $=0.45)$. Step 2 (path "a") examined if CSF KP mediated the effect of plasma KP latent factor. In line with our previous reports [31], plasma KP factor was associated with CSF KP factor via a positive direct path $(\mathrm{Cf}(95 \%$ $\mathrm{Cl})=0.67(0.45,0.88), \quad t=5.57, \quad p<0.001)$. Step 3 (path " $\left.\mathrm{b}^{\prime \prime}\right)$ examined if the association between latent factors representing CSF KP and DMPFC-basal ganglia remained significant after controlling for the effects of plasma KP. connectivity latent) after controlling for plasma KP. CSF KP was negatively associated with DMPFC-basal ganglia connectivity $(\mathrm{Cf}(95 \% \mathrm{Cl})=-0.67(-0.99$, $-0.43), t=4.82, p<0.001)$, after controlling for the effect of plasma KP. Of note, the plasma KP/DMPFC-basal ganglia connectivity association lost its previously noted significance in this step. Step 4 (path $\mathrm{c}^{\prime}$ ) tested if CSF KP completely or partially mediates plasma KP/DMPFC-basal ganglia relationship. CSF KP factor exerted a full-mediation effect on the association between plasma KP and DMPFC-basal ganglia (path $\mathrm{c}^{\prime}, \mathrm{Cf}(95 \% \mathrm{Cl})=-0.44$ $(-0.89,-0.18), t=2.35, p=0.019)$. The full-mediation effect by CSF KP on the association between plasma KP and DMPFC-basal ganglia connectivity had strong predictive power and replicability for both in-sample ( $r$-sq difference $=0.20, f-s q=0.41$ ) and out-ofsample (blindfolding CV test Q-sq difference $=0.22$, $f-s q=0.34$ ) metrics.

\section{Moderation of KP/connectivity associations by immune markers, BMI, and other covariates}

Moderation of above path $c^{\prime}$ by immune markers (TNF/TNFR2), $\mathrm{BMI}$, age, sex, and race were examined. Plasma TNF/TNFR2 was associated with DMPFC-basal ganglia connectivity latent via a negative path $(\mathrm{Cf}(95 \% \mathrm{Cl})=-0.29(-0.55,-0.11), t=2.52, p=$ 0.01). Plasma KP-> CSF KP significantly mediated this association with a strong effect size (mediation effect $\mathrm{Cf}(95 \% \mathrm{Cl})=-0.25$ $(-0.57,-0.09), t=2.02, p=0.043, \mathrm{f}-\mathrm{sq}=0.31)$. A similar negative indirect association between BMI and DMPFC-basal ganglia connectivity was noted $(\mathrm{Cf}(95 \% \mathrm{Cl})=-0.16(-0.39,-0.05), t=$ $2.10, p=0.03)$, which was similarly mediated by Plasma- > CSF KP $(\mathrm{Cf}(95 \% \mathrm{Cl})=-0.16(-0.33,-0.05), t=2.27, p=0.023)$. Age, sex, and race did not exert moderating effects on the above paths. We were unable to compute the effect of obesity upon the path 


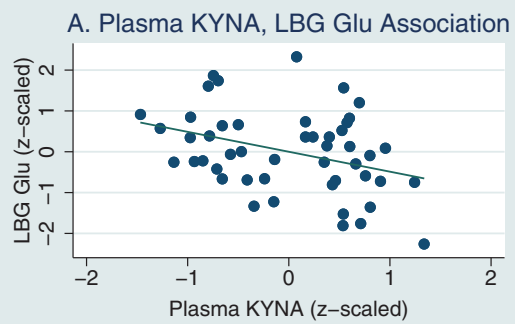

coef $=-0.49$, (robust) $s e=0.17, t=-2.81, p=0.007$

C. Plasma KYNA/QA, LBG Glu Association

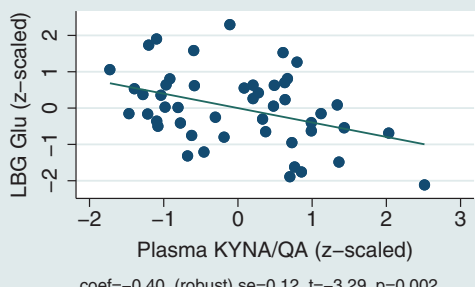

B. Plasma QA, LBG Glu Association

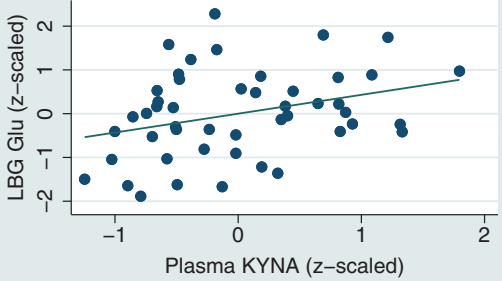

coef $=0.43$, (robust) $s e=0.16, t=2.64, p=0.01$

D. CSF 3HK, LBG Glu Association

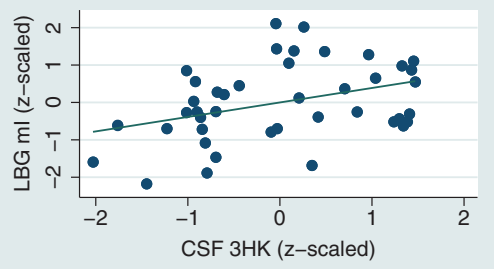

coef $=0.39$, (robust) $s e=0.13, t=3.03, p=0.003$

Fig. 1 KP-MRS associations. The figure represents the partial (A, B) and simple regression plots (C, D) demonstrating the association between kynurenine pathway (KP) metabolites on the $x$-axis and magnetic resonance spectroscopy (MRS)-based markers in the left basal ganglia on the $y$-axis. All values were normalized (using Box-Cox Power Transform) and z-scaled. These are simplified least squares models compared with the more complex lasso regression used in the text. A represents the association between plasma kynurenic acid (KYNA) and left basal ganglia (LBG) glutamate (LBG Glu), B between plasma quinolinic acid (QA) and LBG glutamate (Glu), C between plasma KYNA/QA ratio and LBG Glu, and $\mathbf{D}$ between cerebrospinal fluid (CSF) 3-hydroxykynurenine (3HK) and LBG myo-inositol (LBG ml). The individual coefficients and their significance values are indicated under each graphlet. The voxel used for MRS acquisition (MRS-LBG-VOI) is demonstrated as an inset in Fig. 2A.

model effects due to limitations in the number of subjects with a $\mathrm{BMI}>30)$.

\section{DMPFC-basal ganglia connectivity/anhedonia association}

Anhedonia latent was developed using loading from four items $(\# 8,17,19,22)$ of IDS-SR that clustered together during hierarchical clustering, as shown in Fig. 3. Item \#18: Capacity for Pleasure or Enjoyment was removed following redundancy analysis, as it failed to load adequately in its latent factor (loading coefficient $<0.5)$. Three of 19 connectivities plasma correlated with plasma KYN (DMPFC-DMPFC, DMPFC-left ventrolateral prefrontal region, DMPFC-left dorsolateral prefrontal region), and $1 / 19$ related to CSF 3HK (DMPFC-left basal ganglia) were selected based on their significant loadings onto the path model predicting anhedonia factor (loading coefficient $>0.7$ ). Path and loading coefficients are provided in Supplementary Tables 5-7. Step 1 (Fig. $4 \mathrm{~A})$ examined the direct relationship between KP-associated functional connectivity and anhedonia. A significant negative association was noted between the DMPFC-basal ganglia connectivity factor and the anhedonia factor $(\mathrm{Cf}(95 \% \mathrm{Cl})=-0.56$ $(-0.77,-0.43), t=6.0, p<0.001)$, with a strong effect size ( $r$-sq $=$ 0.36 , Cohen's $\mathrm{f}$-sq $=0.45$ ). Step 2 involved combining all latent factors (plasma KP, CSF KP, DMPFC-basal ganglia connectivity, and anhedonia) within a single unified model, as illustrated in Fig. 4A. A higher-order path model was used to control for high covariance between plasma and CSF KP metabolites. A combined $\mathrm{KP}$ factor including both CSF and plasma KP $(\mathrm{Cf}(95 \% \mathrm{Cl})=0.39$ $(0.28,0.58), t=5.1, p<0.001)$, plasma KP factor $(\mathrm{Cf}(95 \% \mathrm{Cl})=0.27$ $(0.20,0.39), t=5.13, p<0.001)$, and CSF KP factor $(\mathrm{Cf}(95 \% \mathrm{Cl})=$ $0.15(0.01,0.27), t=3.5, p<0.001)$ were all indirectly associated with anhedonia. Intriguingly the path from plasma KP to CSF KP to combined KP factor to DMPFC-basal ganglia connectivity to Anhedonia was significant $(\mathrm{Cf}(95 \% \mathrm{Cl})=0.1(0.05,0.19), t=2.7$, $p=0.007)$.
Associations between MRS metabolites and DMPFC-basal ganglia connectivity: Details of this analysis are presented in Supplementary Information 6 and Supplementary Fig. 4 Power analysis, rigor, and reproducibility. Detailed power estimates and analyses are provided in Supplementary Information 8 and Supplementary Fig. 5. Most of our effect sizes (noted in appropriate sections) were in the strong effect size range with Cohen's f-sq values $>0.25$. With a sample size of 40 , alpha of 0.05 , and power of 0.8 , effect sizes $>0.25$ (for direct paths and linear models) and $>0.2$ (for mediation) were adequate to identify key effects. We have presented data on multiple resampled analyses and sensitivity analyses examining the impact of covariates and disruptive noise factors. The lasso-inferential models used 100 -fold resampling of lasso coefficients, and PLS-SEM used 10,000-fold bootstrapped $\mathrm{Cl}$.

\section{DISCUSSION}

Herein, we report that plasma QA, KYNA, and KYNA/QA were associated with MRS signals of Glu in the left basal ganglia. Plasma KYN/TRP and CSF 3HK were associated with myo-inositol signals in the same region, although this association was subject to moderation by age and BMI. MRS Glu and myo-inositol are believed to index astroglial dysfunction and distress [14, 15]. The precise nature of the astroglial dysfunction in the inflammatory subtype of depression is not yet fully clarified. It is now widely accepted that MRS primarily measures intracellular Glu that exists at much higher concentrations $(5-15 \mathrm{mM})$ compared with the lower concentrations of Glu $(\sim 5-10 \mu \mathrm{M})$ encountered in the extracellular space [74]. A neuronal-astrocytic neurotransmitter cycle exists in the brain in which Glu from the neuronal pool is released into the synaptic cleft as a neurotransmitter, taken up by astrocytes, converted to glutamine, and returned to the neuron in this synaptically inactive form and converted back to Glu $[75,76]$. 


\section{A. Brain Targets of KP Molecules}

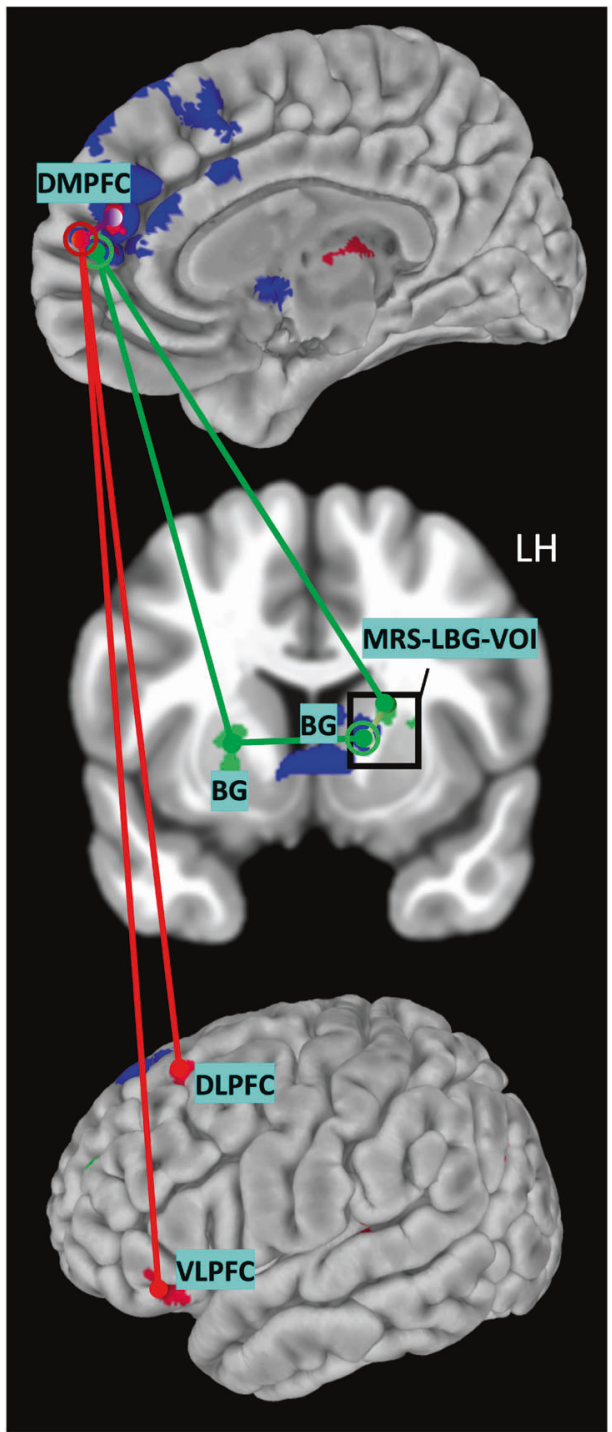

B. DMPFC, BG ReHo Targets of KP System

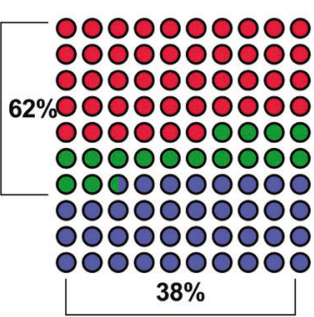

DMPFC

$B G$

Remaining ROIs

\section{DMPFC-BG Connectivity Targets of KP System}

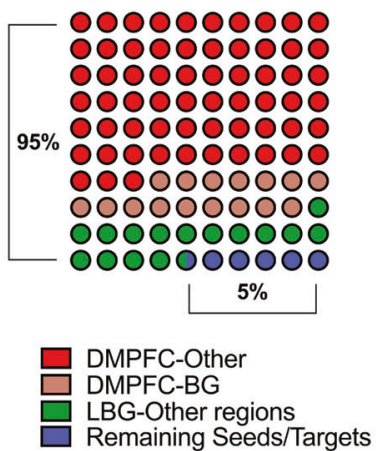

\section{Mediation Models}
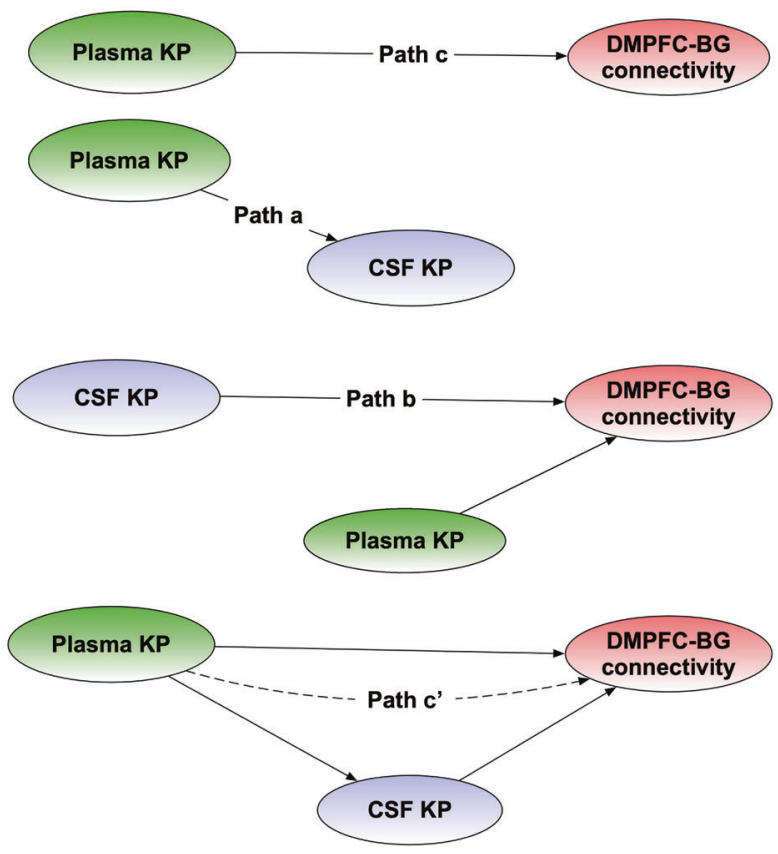

Fig. 2 KP-resting fMRI associations. A presents the primary resting-state functional magnetic resonance imaging ( $r$ MRI) targets of kynurenine pathway (KP) metabolites identified using voxel-based analysis. The areas shaded in deep blue represent the regions of interest (ROI) that demonstrated altered regional homogeneity (ReHo) associated with plasma or cerebrospinal fluid (CSF) KP. Identification of ROIs was based on the correlation between kynurenine (KYN) or 3-hydroxykynurenine (3HK) in either the plasma or CSF. A detailed description of all ReHo seed-ROls thus derived (total $=12$ seeds) is presented in Supplementary Information 4. ROIs correlated with plasma KP are illustrated using red circles and CSF KP-related ROls are illustrated using green circles. These ROls were also set as the seeds in seed-to-whole-brain connectivity analysis. The red or green lines and the red or green uncircled dots represent the connectivity and targets, respectively. Connectivity $z$-scores correlated with plasma and CSF KP measures were derived after controlling for multiple comparisons (threshold $=$ voxel $p<0.001$, cluster $p<0.05$ ). B presents a $10 \times 10$ dot plot depicting the region-wise distribution of voxels (measured using voxel volume $=1$ cubic millimeter/voxel), demonstrating ReHo decreases. As is evident, $62 \%$ of voxels demonstrating decreased ReHo were located either in the dorsomedial prefrontal cortex (DMPFC), left (LBG), or right basal ganglia (RBG) regions, respectively. C demonstrates a similar dot plot demonstrating the distribution of KP-correlated connectivity disruptions between ReHo seed regions and other brain regions. As is evident, $95 \%$ of whole-brain connectivity decreases were associated with DMPFC-BG regions. D represents the overall plan of the path/mediation analyses. Plasma and CSF KP latent factors represent the individual contribution of kynurenine (KYN), 3-hydroxykynurenine (3HK) to each of these factors. DMPFC-basal ganglia (BG) latent factor comprises four different connectivities (DMPFC-LBG, DMPFC-RBG, LBG-LBG, and LBGRBG). Path c examines the relationship between plasma KP and DMPFC-BG connectivity and Path a examines the association between plasma and CSF KP to each other. Path b examines if plasma KP impacts the association between CSF KP and DMPFC-BG connectivity. Path $\mathbf{c}^{\prime}$ presents the model testing mediation by CSF KP of the relationship between plasma KP on DMPFC-BG connectivity.

Neuronal Glu processing by the astroglia is an energy-intensive process involving an expenditure of two molecules of adenosine triphosphate for each molecule of Glu processed [75]. Under mild anesthesia, Glu-glutamine neurotransmitter cycling is believed to consume $>80 \%$ of total glucose oxidation in the cell [75]. However, the energy costs of Glu neurotransmission need to be weighed against the energy demands induced by inflammation. For instance, when immune cells are stimulated in vitro, they consume $\sim 25-30 \%$ more energy, which increases $\sim 60 \%$ during acute infections [77]. Thus, MRS Glu build-up in inflammation may reflect 


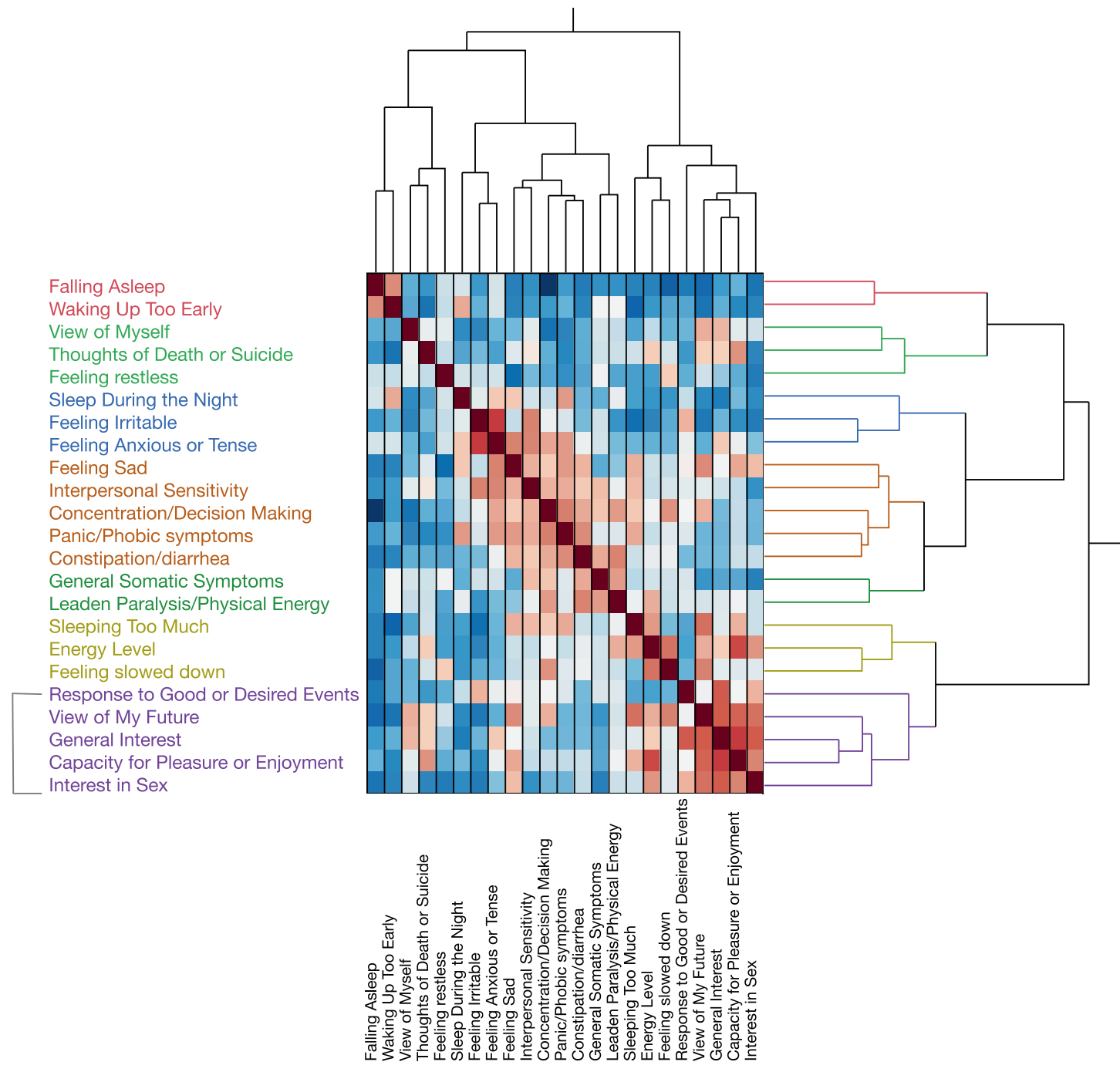

\section{HCA Clustering - Dendrogram}

Fig. 3 Anhedonia-HCA clustering. The figure depicts the dendrogram from hierarchical clustering of individual items on Inventory for Depressive Symptoms-Self Rated Version (IDS-SR). After removing items with ambiguous and unreliable scoring on IDS-SR (details in text), the remaining 23 items were clustered using hierarchical clustering with Ward's agglomerative method. As noted, anhedonia items on IDS-SR (items \#8, 17, 18, 19, and 22) clustered tightly together and were combined into an "Anhedonia" factor. Item \#18: Capacity for Pleasure or Enjoyment was removed during redundancy analysis, as it failed to load adequately onto its latent factor.

the impaired processing capacity resulting from astrocytic distress. MRS Glu increases have been reported in various inflammatory and neuropsychiatric disorders [9]. Similarly, increases in myoinositol signals have been associated with immune pathologies such as multiple sclerosis, human immunodeficiency virus (HIV), and hepatitis $C$ virus infections [16]. Intriguingly, it has also been strongly associated with aging and neurodegenerative dementia [16].

KP metabolism may reflect the convergence of pathologies involving bioenergetic dysfunction, Glu-cycling, and astroglial distress. More recently, the transport of KP molecules across BBB via LAT-1 has been emphasized, as it may be targeted by leucine, a high-affinity blocker of KP transport across LAT-1 [30]. Upon entry via LAT-1, KYN, and 3HK may bind to the NLRP2inflammasome in the astrocytes leading to amplification of neuroinflammatory response via activation of caspase- 1 and nuclear factor kappa-B, culminating in the release of interleukin$1 \mathrm{~b}$ [33]. Thus, KYN may by itself act as a danger-associated molecular pattern that stimulates astrocytic inflammation resulting in increases MRS Glu and myo-inositol. Basal ganglia is a highly vascularized region with many end arteries offering a rich BBB interface for such pathological immune-KP-brain interactions. Increasing inflammation of the glial cells in the basal ganglia region may account for at least some of the reward circuit dysfunction evident in this study.

KP was associated with impaired functional activity and connectivity within/between DMPFC-basal ganglia regions, which in turn was associated with anhedonic items of the IDS-SR. Optogenetic experiments indicate that both the top-down (DMPFC Glu systems) and bottom-up (brainstem dopamine systems) compete for functional dominance over the striatal regions to balance competing motivational demands [78]. While the bottom-up inputs convey prediction error reward signals, the top-down inputs adjust and reorient motivational priorities to facilitate longer-term or non-hedonic objectives. For instance, water (during dehydration) or rest (during inflammation and infection) may be preferred over more powerful, reinforcing hedonic stimuli such as sucrose water [79]. Impairments of DMPFC 


\section{A. Full Path Model}

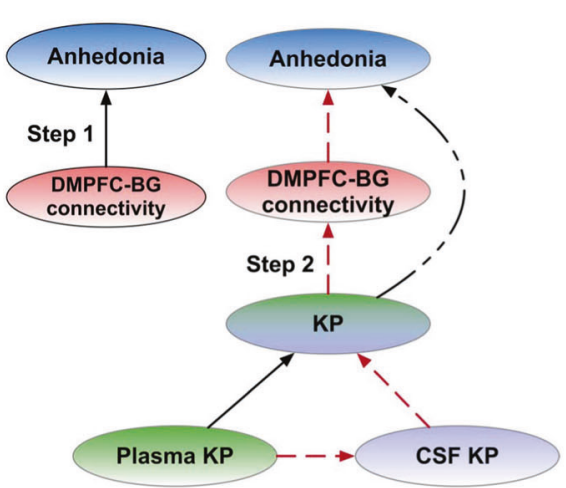

C. Relationship between

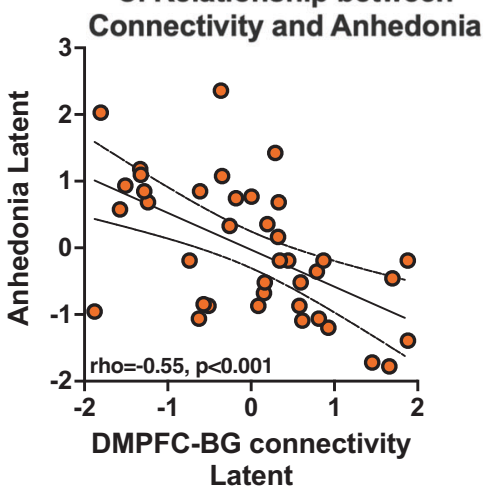

B. Relationship between KP

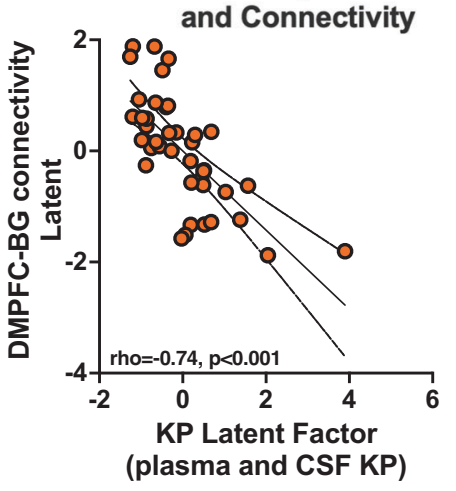

D. Relationship between KP and Anhedonia

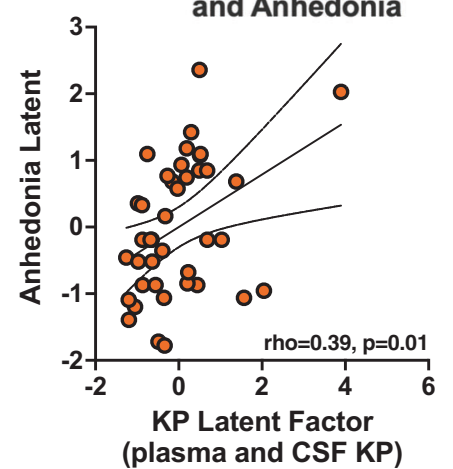

Fig. 4 Connectivity-anhedonia associations. A presents the overall scheme of the path model between all the latent factors within a single, reflective, multilevel path model. Briefly, kynurenine (KYN) and 3-hydroxykynurenine (3HK) in plasma and CSF were loaded onto their respective plasma and CSF kynurenine pathway (KP) factors that loaded further onto a combined KP factor. The KP factor was then related to the dorsomedial prefrontal (DMPFC)-basal ganglia (BG) latent factor that was further linked to the anhedonia latent factor described earlier. DMPFC-BG latent factor was composed of both plasma and CSF connectivities selected based on factor loadings (loading coefficient $>0.7$ ). Three of 4 of the above connectivities, DMPFC-DMPFC (right to left DMPFC), DMPFC-left ventrolateral prefrontal region (LVLPFC), DMPFC-left dorsolateral prefrontal region (DLPFC), were correlated with plasma KYN, and 1/4, i.e., DMPFC-left basal ganglia (LBG) was correlated with CSF $3 \mathrm{HK}$. The red-colored broken lines represent direct paths that combined to create an indirect or mediation effect. Step 1 (A) examined a direct relationship between functional connectivity and anhedonia. B-D represents linear associations between the latent factor scores, with the relationship expressed as Spearman rho values. B represents the direct association between KP and DMPFC-BG connectivity (rho $=0.74, p<$ 0.001 ), and $\mathbf{C}$ presents the direct association between DMPFC-BG connectivity and anhedonia ( $r$ o $=0.55, p<0.001$ ). $\mathbf{D}$ represents the indirect association between KP and anhedonia mediated by DMPFC-BG connectivity (rho $=0.39, p<0.01$ ). A mediation effect of the link between KP and anhedonia by connectivity and the relationship between plasma KP and anhedonia by CSF KP and connectivity was significant (details in text).

might identify a distinct subgroup of depressed patients who may respond to targeted stimulation of this region [80]. DMPFC and its downstream connections have been proposed as promising stimulation targets using transcranial magnetic stimulation for anhedonic depression [81]. Our data build upon this developing literature by demonstrating that KP molecules specifically target the DMPFC-basal ganglia frontostriatal system.

A major thrust of this study is to focus our attention on the anhedonic subtype of depression. Anhedonia refers to a decreased ability to enjoy previously pleasurable experiences [82]. Anhedonia may be viewed as a transdiagnostic entity seen among many neuropsychiatric disorders [6-8]. Our data support conceptualizing anhedonia as a dysfunctional integration between top-down and bottom-up networks that regulate reward functions disrupted by inflammation and its downstream products such as KP molecules [4, 79].

The study's strengths include an in-depth characterization of KP molecules across plasma and CSF compartments, chemical and functional profiling of frontostriatal brain regions, and relating brain changes to anhedonia. However, some caveats will need to be borne in mind. Firstly, our sample lacks a control group, although we intended to examine KP dysfunction specifically among depressed subjects. Induction of anhedonia among healthy subjects by immune stimulation is well known to engage similar top-down and bottom-up brain circuits. Secondly, our data is cross-sectional. Longitudinal or interventional studies will provide more insights into the above-noted interactions. Our sample is relatively small, though the effect sizes were strong enough to support the sample size in our power analysis. Limitations in obtaining reliable measurements for CSF KYNA and QA constrained our ability to examine their association with the imaging outcomes. Nevertheless, future studies are warranted to examine these relationships. The sample was drawn from and represents a limited universe of medication-free, depressed individuals who were willing to undergo MRI scanning and CSF sampling. Our focus on anhedonia may have excluded other depressive symptom dimensions of interest. Suicidality has been significantly related to KP metabolism over several studies. The 
recruitment criteria for the study excluded patients with significant suicidal ideation. Given our focus on frontostriatal systems, we did not examine other brain networks. Of note, biological interpretations of the data are limited by the abstracted nature of resting functional connectivity metrics and the inability of MRS to capture extracellular Glu signals reliably.

In conclusion, the study's findings indicate that KP increases among depressed subjects were associated with greater chemical and functional changes in key hub regions of the frontalsubcortical reward system, leading to an anhedonic depressive subtype. Targeting the peripheral KP system or its transfer into the brain are viable areas of interest. Besides, the effect of KP metabolites on brain chemistry may be mitigated by Glu modulating agents such as ketamine and riluzole. Similarly, the newly emerging field of glial protective agents may help ameliorate the toxic effects of inflammation and KP metabolites on the brain.

\section{REFERENCES}

1. Haroon E, Woolwine BJ, Chen X, Pace TW, Parekh S, Spivey JR, et al. IFN-alphainduced cortical and subcortical glutamate changes assessed by magnetic resonance spectroscopy. Neuropsychopharmacology 2014;39:1777-85.

2. Haroon E, Felger JC, Woolwine BJ, Chen X, Parekh S, Spivey JR, et al. Age-related increases in basal ganglia glutamate are associated with TNF, reduced motivation and decreased psychomotor speed during IFN-alpha treatment: preliminary findings. Brain Behav Immun. 2015;46:17-22.

3. Haroon E, Fleischer CC, Felger JC, Chen X, Woolwine BJ, Patel T, et al. Conceptual convergence: increased inflammation is associated with increased basal ganglia glutamate in patients with major depression. Mol Psychiatry. 2016;21:1351-7.

4. Attwells S, Setiawan E, Rusjan PM, Xu C, Hutton C, Rafiei D, et al. Translocator protein distribution volume predicts reduction of symptoms during open-label trial of celecoxib in major depressive disorder. Biol Psychiatry. 2020;88:649-56.

5. Dantzer R. Role of the kynurenine metabolism pathway in inflammation-induced depression: preclinical approaches. Curr Top Behav Neurosci. 2017;31:117-38.

6. Schwarcz R. The kynurenine pathway of tryptophan degradation as a drug target. Curr Opin Pharmacol. 2004;4:12-17.

7. O'connor JC, André C, Wang Y, Lawson MA, Szegedi SS, Lestage J, et al. Interferon-gamma and tumor necrosis factor-alpha mediate the upregulation of indoleamine 2,3-dioxygenase and the induction of depressive-like behavior in mice in response to bacillus Calmette-Guerin. J Neurosci. 2009;29:4200-9.

8. Guillemin GJ, Kerr SJ, Smythe GA, Smith DG, Kapoor V, Armati PJ, et al. Kynurenine pathway metabolism in human astrocytes: a paradox for neuronal protection. J Neurochem. 2001;78:842-53.

9. Du F, Schmidt W, Okuno E, Kido R, Köhler C, Schwarcz R. Localization of kynurenine aminotransferase immunoreactivity in the rat hippocampus. J Comp Neurol. 1992;321:477-87.

10. Wejksza K, Rzeski W, Okuno E, Kandefer-Szerszen M, Albrecht J, Turski WA Demonstration of kynurenine aminotransferases I and II and characterization of kynurenic acid synthesis in oligodendrocyte cell line (OLN-93). Neurochem Res. 2005;30:963-8.

11. Schwarcz R, Bruno JP, Muchowski PJ, Wu HQ. Kynurenines in the mammalian brain: when physiology meets pathology. Nat Rev Neurosci. 2012;13:465-77.

12. Dantzer R, O'Connor JC, Lawson MA, Kelley KW. Inflammation-associated depression: from serotonin to kynurenine. Psychoneuroendocrinology. 2011;36:426-36.

13. Fukui S, Schwarcz R, Rapoport SI, Takada Y, Smith QR. Blood-brain barrier transport of kynurenines: implications for brain synthesis and metabolism. J Neurochem. 1991;56:2007-17.

14. Walker AK, Wing EE, Banks WA, Dantzer R. Leucine competes with kynurenine for blood-to-brain transport and prevents lipopolysaccharide-induced depressionlike behavior in mice. Mol Psychiatry. 2019;24:1523-32.

15. Haroon E, Welle JR, Woolwine BJ, Goldsmith DR, Baer W, Patel T, et al. Associations among peripheral and central kynurenine pathway metabolites and inflammation in depression. Neuropsychopharmacology. 2020;45:998-1007.

16. Guillemin GJ, Smith DG, Kerr SJ, Smythe GA, Kapoor V, Armati PJ, et al. Characterisation of kynurenine pathway metabolism in human astrocytes and implications in neuropathogenesis. Redox Rep. 2000;5:108-11.

17. Zhang Q, Sun Y, He Z, Xu Y, Li X, Ding J, et al. Kynurenine regulates NLRP2 inflammasome in astrocytes and its implications in depression. Brain Behav Immun. 2020;88:471-81.

18. Matute C, Domercq M, Sanchez-Gomez MV. Glutamate-mediated glial injury: mechanisms and clinical importance. Glia. 2006;53:212-24.
19. Schwarcz R. Kynurenines and glutamate: multiple links and therapeutic implications. Adv Pharmacol. 2016;76:13-37.

20. Guillemin GJ. Quinolinic acid, the inescapable neurotoxin. FEBS J. 2012;279:1356-65

21. Piani D, Fontana A. Involvement of the cystine transport system xc- in the macrophage-induced glutamate-dependent cytotoxicity to neurons. J Immunol. 1994;152:3578-85.

22. Dantzer R, Walker AK. Is there a role for glutamate-mediated excitotoxicity in inflammation-induced depression? J Neural Transm. 2014;121:925-32.

23. McCullumsmith RE, Sanacora G. Regulation of extrasynaptic glutamate levels as a pathophysiological mechanism in disorders of motivation and addiction. Neuropsychopharmacology. 2015;40:254-5.

24. Dorsett CR, McGuire JL, DePasquale EA, Gardner AE, Floyd CL, McCullumsmith RE. Glutamate neurotransmission in rodent models of traumatic brain injury. J Neurotrauma. 2017;34:263-72.

25. Hardingham GE, Bading $H$. Synaptic versus extrasynaptic NMDA receptor signalling: implications for neurodegenerative disorders. Nat Rev Neurosci. 2010;11:682-96.

26. Hardingham GE, Fukunaga $Y$, Bading $H$. Extrasynaptic NMDARs oppose synaptic NMDARs by triggering CREB shut-off and cell death pathways. Nat Neurosci. 2002;5:405-14.

27. Okubo $Y$, lino $M$. Visualization of glutamate as a volume transmitter. J Physiol. 2011;589:481-8.

28. Sykova E. Extrasynaptic volume transmission and diffusion parameters of the extracellular space. Neuroscience. 2004;129:861-76.

29. Haroon E, Chen X, Li Z, Patel T, Woolwine BJ, Hu XP, et al. Increased inflammation and brain glutamate define a subtype of depression with decreased regional homogeneity, impaired network integrity, and anhedonia. Transl Psychiatry. 2018;8:189.

30. Biswal BB, Van Kylen J, Hyde JS. Simultaneous assessment of flow and BOLD signals in resting-state functional connectivity maps. NMR Biomed. 1997;10:165-70.

31. Bluhm RL, Miller J, Lanius RA, Osuch EA, Boksman K, Neufeld RW, et al. Spontaneous low-frequency fluctuations in the BOLD signal in schizophrenic patients: anomalies in the default network. Schizophr Bull. 2007;33:1004-12.

32. Kannurpatti SS, Biswal BB. Detection and scaling of task-induced fMRI-BOLD response using resting state fluctuations. Neuroimage. 2008;40:1567-74.

33. Lowe MJ, Phillips MD, Lurito JT, Mattson D, Dzemidzic M, Mathews VP. Multiple sclerosis: low-frequency temporal blood oxygen level-dependent fluctuations indicate reduced functional connectivity initial results. Radiology. 2002:224:184-92.

34. Cornwell BR, Salvadore G, Furey M, Marquardt CA, Brutsche NE, Grillon C, et al. Synaptic potentiation is critical for rapid antidepressant response to ketamine in treatment-resistant major depression. Biol Psychiatry. 2012;72:555-61.

35. Hoptman MJ, Zuo XN, Butler PD, Javitt DC, D'Angelo D, Mauro CJ, et al. Amplitude of low-frequency oscillations in schizophrenia: a resting state fMRI study. Schizophr Res. 2010;117:13-20.

36. Ongür D, Lundy M, Greenhouse I, Shinn AK, Menon V, Cohen BM, et al. Default mode network abnormalities in bipolar disorder and schizophrenia. Psychiatry Res. 2010;183:59-68.

37. Zuo XN, Di Martino A, Kelly C, Shehzad ZE, Gee DG, Klein DF, et al. The oscillating brain: complex and reliable. Neuroimage. 2010;49:1432-45.

38. Raison CL, Dantzer R, Kelley KW, Lawson MA, Woolwine BJ, Vogt G, et al. CSF concentrations of brain tryptophan and kynurenines during immune stimulation with IFN-alpha: relationship to CNS immune responses and depression. Mol Psychiatry. 2010;15:393-403.

39. Capuron L, Neurauter G, Musselman DL, Lawson DH, Nemeroff CB, Fuchs D, et al. Interferon-alpha-induced changes in tryptophan metabolism. relationship to depression and paroxetine treatment. Biol Psychiatry. 2003;54:906-14.

40. Bay-Richter C, Linderholm KR, Lim CK, Samuelsson M, Träskman-Bendz L, Guillemin GJ, et al. A role for inflammatory metabolites as modulators of the glutamate $\mathrm{N}$-methyl-D-aspartate receptor in depression and suicidality. Brain Behav Immun. 2015:43:110-7.

41. Erhardt S, Lim CK, Linderholm KR, Janelidze S, Lindqvist D, Samuelsson M, et al. Connecting inflammation with glutamate agonism in suicidality. Neuropsychopharmacology. 2013;38:743-52.

42. Savitz J, Dantzer R, Meier TB, Wurfel BE, Victor TA, Mclntosh SA, et al. Activation of the kynurenine pathway is associated with striatal volume in major depressive disorder. Psychoneuroendocrinology. 2015;62:54-58.

43. Parrott JM, Redus L, Santana-Coelho D, Morales J, Gao X, O'Connor JC. Neurotoxic kynurenine metabolism is increased in the dorsal hippocampus and drives distinct depressive behaviors during inflammation. Transl Psychiatry. 2016;6:e918.

44. Walker AK, Budac DP, Bisulco S, Lee AW, Smith RA, Beenders B, et al. NMDA receptor blockade by ketamine abrogates lipopolysaccharide-induced depressive-like behavior in $\mathrm{C} 57 \mathrm{BL} / 6 \mathrm{~J}$ mice. Neuropsychopharmacology. 2013:38:1609-16. 
45. Dantzer R, O'Connor JC, Freund GG, Johnson RW, Kelley KW. From inflammation to sickness and depression: when the immune system subjugates the brain. Nat Rev Neurosci. 2008;9:46-56.

46. O'connor JC, Lawson MA, André C, Briley EM, Szegedi SS, Lestage J, et al. Induction of IDO by bacille Calmette-Guerin is responsible for development of murine depressive-like behavior. J Immunol. 2009;182:3202-12.

47. First MB, Spitzer RL, Gibbon M, Williams JB. Structured clinical interview for DSMIV. Washington DC: American Psychiatric Press; 1997.

48. Hamilton M. A rating scale for depression. J Neurol Neurosurg Psychiatry. 1960;23:56-62.

49. Felger JC, Li Z, Haroon E, Woolwine BJ, Jung MY, Hu X, et al. Inflammation is associated with decreased functional connectivity within corticostriatal reward circuitry in depression. Mol Psychiatry. 2016;21:1358-65.

50. Rush AJ, Gullion CM, Basco MR, Jarrett RB, Trivedi MH. The inventory of depressive symptomatology (IDS): psychometric properties. Psychol Med. 1996;26:477-86.

51. Trivedi MH, Rush AJ, Ibrahim HM, Carmody TJ, Biggs MM, Suppes $T$, et al. The inventory of depressive symptomatology, clinician rating (IDS-C) and self-report (IDS-SR), and the quick inventory of depressive symptomatology, clinician rating (QIDS-C) and self-report (QIDS-SR) in public sector patients with mood disorders: a psychometric evaluation. Psychol Med. 2004;34:73-82.

52. Felger JC, Haroon E, Patel TA, Goldsmith DR, Wommack EC, Woolwine BJ, et al. What does plasma CRP tell us about peripheral and central inflammation in depression? Mol Psychiatry. 2020;25:1301-11.

53. Cox RW, Chen G, Glen DR, Reynolds RC, Taylor PA. FMRI clustering in AFNI: falsepositive rates redux. Brain Connect. 2017;7:152-71.

54. Eklund $A$, Nichols TE, Knutsson $H$. Cluster failure: why fMRI inferences for spatial extent have inflated false-positive rates. Proc Natl Acad Sci USA. 2016;113:7900-5.

55. Ringle CM, Sven W, Becker J-M. "SmartPLS". Boenningstedt: SmartPLS GmbH. 2015. Available from: http://www.smartpls.com.

56. Baron RM, Kenny DA. The moderator-mediator variable distinction in social psychological research: conceptual, strategic, and statistical considerations. J Pers Soc Psychol. 1986;51:1173-82.

57. Brand A, Richter-Landsberg C, Leibfritz D, Multinuclear NMR. studies on the energy metabolism of glial and neuronal cells. Dev Neurosci. 1993;15:289-98.

58. Brand A, Leibfritz D, Richter-Landsberg C. Oxidative stress-induced metabolic alterations in rat brain astrocytes studied by multinuclear NMR spectroscopy. J Neurosci Res. 1999;58:576-85.

59. Torres-Platas SG, Hercher C, Davoli MA, Maussion G, Labonté B, Turecki G, et al. Astrocytic hypertrophy in anterior cingulate white matter of depressed suicides. Neuropsychopharmacology. 2011;36:2650-8.

60. Zhang X, Alnafisah RS, Hamoud A-RA, Shukla R, Wen Z, McCullumsmith RE, et al. Role of Astrocytes in Major Neuropsychiatric Disorders. Neurochem Res. (2021). Online ahead of print. https://doi.org/10.1007/s11064-020-03212-x.

61. Stagg CJ, Rothman DL. Magentic resonance spectroscopy - Tools for neuroscience research and emerging clinical applications. London: Academic; 2014.

62. Sibson NR, Dhankhar A, Mason GF, Rothman DL, Behar KL, Shulman RG. Stoichiometric coupling of brain glucose metabolism and glutamatergic neuronal activity. Proc Natl Acad Sci USA. 1998;95:316-21.

63. Zhou Y, Danbolt NC. Glutamate as a neurotransmitter in the healthy brain. J Neural Transm. 2014;121:799-817.

64. Straub RH. The brain and immune system prompt energy shortage in chronic inflammation and ageing. Nat Rev Rheumatol. 2017;13:743-51.

65. Haroon E, Miller AH, Sanacora G. Inflammation, glutamate, and glia: a trio of trouble in mood disorders. Neuropsychopharmacology. 2017;42:193-215.

66. Quarantelli M. MRI/MRS in neuroinflammation: methodology and applications. Clin Transl Imaging. 2015;3:475-89.

67. Ferenczi EA, Zalocusky KA, Liston C, Grosenick L, Warden MR, Amatya D, et al. Prefrontal cortical regulation of brainwide circuit dynamics and reward-related behavior. Science. 2016;351:aac9698.

68. Treadway MT, Cooper JA, Miller AH. Can't or won't? Immunometabolic constraints on dopaminergic drive. Trends Cogn Sci. 2019;23:435-48.

69. Drysdale AT, Grosenick L, Downar J, Dunlop K, Mansouri F, Meng Y, et al. Restingstate connectivity biomarkers define neurophysiological subtypes of depression. Nat Med. 2017;23:28-38.

70. Dunlop K, Talishinsky A, Liston C. Intrinsic brain network biomarkers of antidepressant response: a review. Curr Psychiatry Rep. 2019;21:87.

71. Buch $A M$, Liston C. Dissecting diagnostic heterogeneity in depression by integrating neuroimaging and genetics. Neuropsychopharmacology. 2021;46:156-75.

72. Downar J, Geraci J, Salomons TV, Dunlop K, Wheeler S, McAndrews MP, et al. Anhedonia and reward-circuit connectivity distinguish nonresponders from responders to dorsomedial prefrontal repetitive transcranial magnetic stimulation in major depression. Biol Psychiatry. 2014;76:176-85.
73. American Psychiatric Association. Diagnostic and statistical manual of mental disorders-V. 5th ed. Washington, DC: American Psychiatric Press; 2013.

74. Kaiser RH, Andrews-Hanna JR, Wager TD, Pizzagalli DA. Large-scale network dysfunction in major depressive disorder: a meta-analysis of resting-state functional connectivity. JAMA Psychiatry. 2015;72:603-11.

75. Cooper JA, Arulpragasam AR, Treadway MT. Anhedonia in depression: biological mechanisms and computational models. Curr Opin Behav Sci. 2018;22:128-35.

76. Rizvi SJ, Pizzagalli DA, Sproule BA, Kennedy SH. Assessing anhedonia in depression: potentials and pitfalls. Neurosci Biobehav Rev. 2016;65:21-35.

77. Lucido MJ, Bekhbat M, Goldsmith DR, Treadway MT, Haroon E, Felger JC, et al. Aiding and abetting anhedonia: impact of inflammation on the brain and pharmacologic implications. Pharm Rev. 2021 Jul;73:1084-117.

78. Covinsky KE, Cenzer IS, Yaffe K, O'Brien S, Blazer DG. Dysphoria and anhedonia as risk factors for disability or death in older persons: implications for the assessment of geriatric depression. Am J Geriatr Psychiatry. 2014;22:606-13.

79. Eisenberger NI, Berkman ET, Inagaki TK, Rameson LT, Mashal NM, Irwin MR. Inflammation-induced anhedonia: endotoxin reduces ventral striatum responses to reward. Biol Psychiatry. 2010;68:748-54.

80. Harrison NA, Brydon L, Walker C, Gray MA, Steptoe A, Critchley HD. Inflammation causes mood changes through alterations in subgenual cingulate activity and mesolimbic connectivity. Biol Psychiatry. 2009;66:407-14.

81. Harrison NA, Cooper E, Dowell NG, Keramida G, Voon V, Critchley HD, et al. Quantitative magnetization transfer imaging as a biomarker for effects of systemic inflammation on the brain. Biol Psychiatry. 2015;78:49-57.

82. Krishnadas R, Harrison NA. Depression phenotype, inflammation, and the brain: implications for future research. Psychosom Med. 2016;78:384-8.

\section{ACKNOWLEDGEMENTS}

This study was supported by grants R01MH087604 (A.H.M.), R01MH107033 (E.H.), K23MH091254 (E.H.), R01MH112076 (A.H.M. and E.H.), R01MH109637(J.C.F.), R21MH121891 (J.C.F. and A.H.M.), and R61MH121625 (J.C.F.) from the National Institute of Mental Health; UL1TR002378 (Georgia Clinical and Translational Alliance, Georgia (TSA) from the National Center for Advancing Translational Sciences, and by a Shared Instrumentation Grant (S10) grant S100D016413-01 to the Emory University Center for Systems Imaging Core. The content is solely the responsibility of the authors and does not necessarily represent the official views of the National Institutes of Health. The funding sources had no role in the design and conduct of the study; collection, management, analysis, and interpretation of the data; preparation, review, or approval of the manuscript; and decision to submit the manuscript for publication. We wish to thank Predictum, Inc (https://predictum.com/products/svem/) for providing free access to a trial version of the SVEM Software Module, used for analysis described in Supplementary Information 6.

\section{AUTHOR CONTRIBUTIONS}

X.C., E.H., J.C.F., and A.H.M. participated in data analysis and preparation of the paper. X.C. was blinded to clinical data and handled all scan analyses. B.J.W. conducted all clinical assessments and rating instruments but was blind to scan analyses. J.C.F. supervised all biological assays and supervised shipping of analytes to outside vendors and receipt of completed data. W.B., D.J.B., and V.D.T. participated in data collection, uploading to database, and following up with subjects. T.P. conducted all LPs.

\section{COMPETING INTERESTS}

Dr. Miller is a paid consultant to Boehringer Ingelheim. The remaining authors declare no competing interests.

\section{ADDITIONAL INFORMATION}

Supplementary information The online version contains supplementary material available at https://doi.org/10.1038/s41398-021-01587-1.

Correspondence and requests for materials should be addressed to Ebrahim Haroon.

Reprints and permission information is available at http://www.nature.com/ reprints

Publisher's note Springer Nature remains neutral with regard to jurisdictional claims in published maps and institutional affiliations. 
Open Access This article is licensed under a Creative Commons Ac Attribution 4.0 International License, which permits use, sharing,
adaptation, distribution and reproduction in any medium or format, as long as you give appropriate credit to the original author(s) and the source, provide a link to the Creative Commons license, and indicate if changes were made. The images or other third party material in this article are included in the article's Creative Commons license, unless indicated otherwise in a credit line to the material. If material is not included in the article's Creative Commons license and your intended use is not permitted by statutory regulation or exceeds the permitted use, you will need to obtain permission directly from the copyright holder. To view a copy of this license, visit http://creativecommons. org/licenses/by/4.0/.

(c) The Author(s) 2021 